See discussions, stats, and author profiles for this publication at: https://www.researchgate.net/publication/319181903

\title{
Assessing the effects of a relational training intervention on fluid intelligence among a sample of socially disadvantaged children in Bangladesh
}

Article in The Psychological record · October 2017

Dol: 10.1007/540732-018-0273-4

\section{CITATIONS}

2 authors:

Micah Amd

University of the South Pacific

18 PUBLICATIONS 73 CITATIONS

SEE PROFILE
READS

946

Bryan Roche

National University of Ireland, Maynooth

131 PUBLICATIONS 4,864 CITATIONS

SEE PROFILE

Some of the authors of this publication are also working on these related projects:

Project The effect of varying stimulus relatedness in implicit measures View project

Project Behavioral and Brain Mechanisms of Avoidance View project 


\title{
Assessing the Effects of a Relational Training Intervention on Fluid Intelligence Among a Sample of Socially Disadvantaged Children in Bangladesh
}

\author{
Micah Amd ${ }^{1,2,3}$ (1) Bryan Roche ${ }^{2}$ \\ (C) Association for Behavior Analysis International 2018
}

\begin{abstract}
A growing consensus is that performances on standardized intelligence tests can be positively influenced by interventions that focus on improving relational reasoning. One such intervention, known as SMART (Strengthening Mental Abilities with Relational Training), consists of presenting participants with increasingly complex exemplars of relational reasoning tasks involving premises associated along same-as, opposite-of, more-than, and less-than relations. Following multiple training and testing sessions involving these relations, increased fluency in SMART is related to enhanced performances on tests of intellectual ability (Cassidy et al., 2016). The current study expands upon previous investigations on SMART in two ways. First, we explored whether the amount of training undertaken can predict changes in intelligence test performances. Second, we assessed whether SMART training could be effective for a non-English speaking, socioeconomically disadvantaged cohort. Changes in intelligence were assessed via administrations of the standard Raven's Progressive Matrices before and after SMART training. Our results show that the stage of SMART training completed is positively related to changes in Ravens' performances and that such training can be effective for non-English cohorts.
\end{abstract}

Keywords Relational reasoning $\cdot$ Intelligence $\cdot$ Applied learning theory

Intelligence as variously measured is more malleable than previously thought (Jäeggi, Buschkuehl, Jonides, \& Shah, 2011; Sternberg, 2008), with a host of recent studies claiming to have identified means of increasing intellectual ability (e.g., Cassidy et al., 2016; Cassidy, Roche, \& Hayes, 2011; Hayes \& Stewart, 2016; Jäeggi et al., 2011, but see Burns \& Staats, 1981). Among behaviorally oriented psychologists, the assumption that intelligence tests measure skills that are more likely learned than innate is not new (Staats, 1971), with a number of interventions reporting some degree of intellectual gains over the years (Lovaas, 1987; Sternberg, 1988). More recently, a relational training program called SMART

\section{Micah Amd}

micah.amd.eab@hotmail.com

Federal University of Sao Carlos, Sao Carlos, Brazil

2 Maynooth University, Dublin, Ireland

3 McGill University, Montreal, Canada
(Strengthening Mental Abilities through Relational Training; Cassidy et al., 2016) has been applied with some success in enhancing intellectual abilities (Hayes \& Stewart, 2016; Thirus, Starbrink, \& Jansson, 2016). SMART involves presenting participants with increasingly complex relational propositions across time-restricted trials, with participants required to derive whether a proposition constructed from the premises provided is accurate or not (Fig. 1). SMART was developed within the framework of relational frame theory (RFT; Hayes, Barnes-Holmes, \& Roche, 2001), an account of verbal learning and cognition (for a general introduction, see Blackledge, 2003). According to RFT, the act of deriving relations between arbitrarily (socially) defined events in the presence of particular contextual cues is fundamental in human cognition (Amd \& Roche, 2016, p. 14). It follows that if relating skills are fundamental for intellectual performance (cf., Roche, Cassidy, \& Stewart, 2013), then programs which focus on developing relating behaviors should enhance intellectual performance (Cassidy et al., 2016). The SMART procedure involves presenting propositions along relations of 


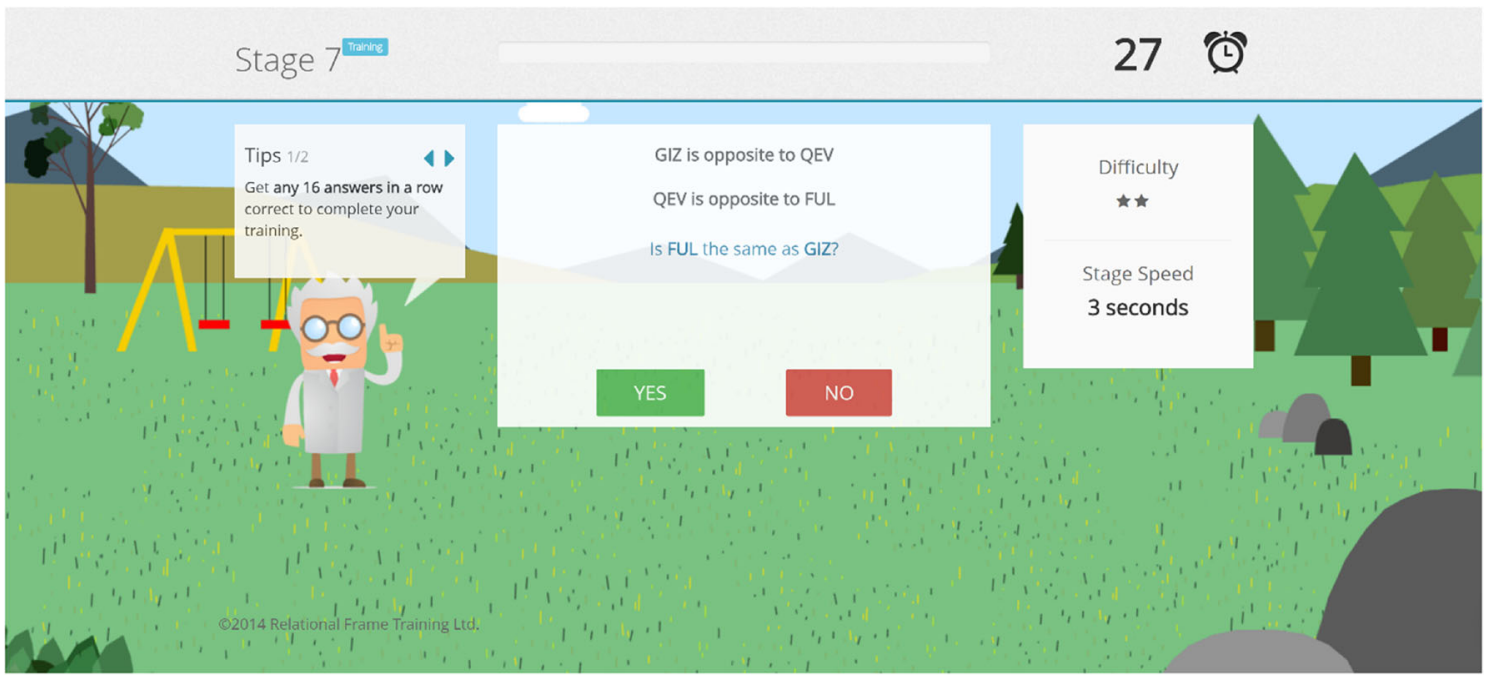

Fig. 1 Sample screen from the SMART program used here (www. raiseyourIQ.com). The numbers on the top left and right sides indicate stage number (7) and the remaining time ( $29 \mathrm{~s})$ within which to respond.
The timer reset to $30 \mathrm{~s}$ following a response. The left-right positioning of the two response options (Yes, No) were counter-balanced across trials equivalence ('same as'), comparison ('more than, less than'), and opposition, independently or in combination (see Cassidy et al., 2011 for details). There is increasing evidence that relating along these dimensions are vital for verbal reasoning and problem-solving (Dixon, Whiting, Rowsey, \& Belisly, 2014; Moran, Stewart, McElwee, \& Ming, 2010; Roche et al., 2013). Furthermore, increased relational fluency has been positively correlated with gains in standardized intelligence quotient (IQ) scores (O'Toole, Barnes-Holmes, Murphy, O'Connor, \& Barnes-Holmes, 2009). These findings suggest that 'relating' per se may constitute a basic behavioral repertoire on which more complex intellectual skills can be learned (Staats, 2012, p. 178). This can explain how improving relating skills vis-à-vis SMART training can improve performances on standard measures of intelligence (Cassidy et al., 2016; Hayes \& Stewart, 2016; Roche et al., 2013; Thirus et al., 2016). A brief description of this procedure is provided below. In a typical SMART procedure, participants can undergo up to 55 stages of training and testing involving relational reasoning tasks under strict speed and accuracy criteria. Successive blocks of training and testing stages become increasingly more complex in terms of the number and type of relations presented (Fig. 1). The goal in SMART training is to bring the participant's responding under control of relational contextual cues rather than the formal features of the stimuli (McIlvane \& Dube, 2003). Each stage involves presenting between one and three relational propositions along dimensions of more, less, more-less combined, same, opposite, and same-opposite combined, with each proposition containing nonsense word stimuli conjoined by a meaningful relational cue (i.e., the words SAME-AS). The propositions are followed by a question asking whether a non-explicit (inferred) relation between the stimuli is correct by clicking on the words YES or NO on the screen (Fig. 1). Corrective feedback is provided after each response. Trial-by-trial corrective feedback is offered until participants produce the response criterion of 16 consecutively correct responses on each of the 55 stages of increasing relational complexity. This is followed by a testing stage involving relation types similar to those presented in the preceding training stage. Participants have to produce 16 consecutively correct responses without any feedback during tests. Meeting this criterion advances participants to the next stage of relational complexity; failure to do so recycles the participant to the preceding training stage. Investigations on the effects of SMART on intellectual ability generally consist of participants completing the prescribed 55 training stages, sometimes across several months (Cassidy et al., 2016; Hayes \& Stewart, 2016, Thirus et al., 2016; for variations on this procedure, see Parra \& Ruiz, 2016; Vizcaino-Torres et al., 2015). A general observation across those studies was a significant gain in intellectual ability following training completion, as measured by the Wechsler Intelligence Scales (Cassidy et al., 2011) or Raven's Progressive Matrices (Thirus et al., 2016), suggesting a positive correlation between relational training and intellectual gains (Roche et al., 2013; Staats, 2012). This raises the question as to whether different 'doses' of relational training can predict subsequent improvements in intellectual ability, which no previously published investigation has shown. This is important because in the first half (stages 1-29) of SMART training, participants view only same and opposite relations, whereas in the second half (stages 30-55), participants view only more and less relations. Across both same/opposite (129) and more/less (33-55) progressions, the first few stages consist of simple relations that school-age children are likely to be familiar with already. These relations gradually increase 
in complexity across each half. For example, stage 1 would be simpler than stage 15 , which would be simpler than stage 29 , only for same, opposite, and same-opposite combined relations. Similarly, more/less relations would increase in complexity from stage 30 to stage 55 . Delineating the dosage effects from various stages in training can be used to determine whether relation type (e.g., same/opposite) and/or complexity (1-node/2-node) differentially impacts intellectual ability (Cassidy, Roche, \& O'Hora, 2010). Some evidence for potential dosage effects from the number of SMART stages completed on intellectual enhancement is provided by Thirus et al. (2016). Although those researchers did not explore for dosage effects per se, the data for participants who had failed to complete the training, when compared to those who had completed training and those who did not, imply that the last SMART stage completed may predict rises in intellectual ability. Specifically, collating participants who failed the training in its entirety with those who completed training reduced, but did not eliminate, statistically significant differences in IQ gains when compared against the matched controls. A primary goal of the current study therefore was to determine whether the last stage completed in SMART training could predict changes in intellectual ability. A secondary goal involved asking whether SMART training could be effective for non-English speaking children from austere backgrounds. Specifically, the cohort under investigation presently consisted of non-English speaking socio-economically disadvantaged Bengali children in sub-optimal conditions. The outcomes will be used to inform the development of SMART as a low cost, accessible, and automated behavioral intervention for populations with limited resources (e.g., Ainscow, 2005). In sum, we explored whether SMART training could enhance the intellectual skills of a cohort of disadvantaged children who casually attend a non-profit charitable status school in Dhaka, Bangladesh. Changes in intellectual ability were inferred from pre-SMART and post-SMART assessments with the standard Raven's Progressive Matrices (sRPM; Raven \& Court, 1993). The sRPM was selected due to its cultural non-specificity, lack of reliance on literacy, and its validity as a proxy of fluid intelligence (Carpenter, Just, \& Shell, 1990; Cattell, 1963). There is growing evidence that sRPM performances may be indicative of general intelligence as well (e.g., Bakhiet, Haseeb, Seddieg, Cheng, \& Lynn, 2015; Sefcek, Miller, \& Figueredo, 2016; te Nijenhuis et al., 2016). Briefly, fluid intelligence (Gf) refers to the capacities involved in analyzing and resolving novel problems and contributes to educational performance (Adey, Csapó, Demetriou, Hautamäki, \& Shayer, 2007), scholastic success (Rohde \& Thompson, 2007), and predicts both career choice (Di Fabio \& Saklofske, 2014) and career success (Sternberg, 2008). Consequently, the SRPM may be considered a reliable proxy for IQ measurements (e.g., see Daley, Whaley, Sigman, Espinosa, \& Neumann, 2003, but also see Hayes, Petrov, \&
Sederberg, 2015). To address our question of dosage effects, it was decided $a b$ intio that no participants would be excluded from the study due to failure to complete the intervention training program. Our hypothesis was that the degree of training undertaken during a fixed ( 3 months) training period would be related to gains in the sRPM post-intervention. Given the backgrounds of the children involved and the context more generally, we did not expect that the SMART intervention would be completed by the majority of participants.

\section{Method}

\section{Participants}

Fifty-two children from a small school in Dhaka, Bangladesh, aged between 6 and 14 years, were initially selected for the present study. Following recruitment, political turmoil in the region (Ruud, 2015) disrupted the start of training and led to four children failing to return to school. The data for a further eight children were lost due to technical and administrative errors. Finally, five children were discovered to have shared login codes for the online training system; hence, their data was considered unreliable and removed from the data set. This left a final sample of 35 children (16 females, mean age = 10 years, $S D=1.92 ; 19$ males, mean age $=9$ years, $S D=$ 1.57) for the remainder of the study. The study described was approved by the local school board, which functioned as the ethics committee for the work reported here. In accordance with their suggestions, two teachers accompanied the researcher at all times during data collection. The current research complied with the ethical and child protection guidelines set forth by the Federal University of Sao Carlos.

\section{Materials}

\section{Raven's Matrices}

The sRPM (Raven, 2000) consists of 60 equally weighted test items. Each item depicts a series of two-dimensional figures with the final part of the series missing. The participant's task is to select from an array of component figures the 'correct' component to complete the series. Scores are calculated by summating correct responses in the range of 0-60 and can provide percentile rankings based on age-adjusted norms (Raven, 2000). In the present design, participants were provided $60 \mathrm{~min}$ to complete the task. No participant required more than $40 \mathrm{~min}$ to complete the sRPM.

The sRPM is a non-verbal task consisting of visual analogy problems (Carpenter et al., 1990) that can be administered to populations from non-English backgrounds (Moran, 1986; Owen, 1992; Raven \& Court, 1993; Valencia, 1984). The 
visual nature of the SRPM means lesser reliance on culture-specific knowledge in comparison to many mainstream intelligence scales (Cockcroft, Alloway, Copello, \& Milligan, 2015; Miele, 1979). The Ravens' Matrices is positively correlated with mainstream intelligence tests (e.g., $>0.75$, Owen, 1992), is internally reliable (e.g., $>0.8$, Raven, 2000), and is considered a valid proxy for full scale intelligence (Bakhiet et al., 2015; Sefcek et al., 2016; te Nijenhuis et al., 2016). The sRPM also displays good test-retest reliability (e.g., > 0.8, Raven, 2000).

\section{Strengthening Mental Abilities with Relational Training}

SMART training involves presenting participants with propositions consisting of the relations of same, opposite, same-opposite combined, more, less and more-less combined, followed by a query asking whether a specific relation is inferable from the premises provided. Specifically, out of 55 stages in total, the first 29 involve training along same, opposite, and same-opposite combined relations; the second 26 stages involve training along more, less, and more-less combined relations. For example, a combined same-opposite training trial might involve the presentation of the statements 'WUG is the same as JEZ' and 'JEZ is the opposite of SIG' followed by the question, 'Is WUG the same as SIG?' Note that these 3-character nonsense words are randomly generated between and across trials. Participants can select between Yes and No response options that appear in counter-balanced order on the bottom left and right sides of the screen within $30 \mathrm{~s}$ of trial onset (Fig. 1). Participants were provided trial-by-trial feedback until 16 consecutively correct responses were made (training). Participants then had to produce 16 consecutively correct responses again, but without any corrective feedback. Failing to do so resulted in the participant being recycled to the preceding training stage. The training and testing cycle continued ad infinitum for each stage until the test criterion was reached (16 consecutively correct responses without feedback). The SMART procedure has been used in previous investigations into the effects of relational training on intellectual ability (www.raiseyourIQ.com) and is presently available commercially. ${ }^{1}$

\section{Relational Ability Index}

Prior to commencing the SMART procedure described above, but after English instruction training, ${ }^{2}$ participants underwent

\footnotetext{
${ }^{1}$ The second author is part owner of www.RaiseYourIQ.com, which commercially provides SMART training. We have reported this conflict of interest to the editor.

${ }^{2}$ Our cohort was unfamiliar with English and had to be trained along the meaning of the relational terms 'More, Less, Same' and 'Opposite', as well as the feedback terms 'Correct' and 'Wrong'.
}

a brief test of relational abilities known as the Relational Abilities Index (RAI; see Cassidy et al., 2016). The RAI consists of 55 relational questions presented without feedback. These correspond with the 55 stages of complexity in the SMART procedure. The RAI and sRPM performances served as a baseline measure of relational responding ability that allowed for subsequent correlational analyses.

\section{Design}

A multiple linear regression with four predictors (age, baseline performances on the sRPM and RAI, number of stages completed) was employed to explain variances in SRPM performances before and after SMART training (post-sRPM-baseline sRPM). We ran a prospective 2-tailed power analysis (Faul, Erdfelder, Lang, \& Buchner, 2007), with H1 $\rho^{2}$ determined to be .498 and correlation coefficients of four predictor variables (age, sRPM baseline, RAI baseline, last stage completed) set to $-0.100,-0.454,0.006$, and 0.312 , respectively, based on the data reported by Thirus et al. (Thirus et al., 2016, p.151). Holding the possibility of a type 1 error $(\alpha)$ to be 0.05 , power $(1-\beta)$ to be 0.9 and $\mathrm{H} 0 \rho^{2}$ set at 0 , we found that there is a $91 \%$ chance of correctly rejecting the $\mathrm{H} 0$ where the multiple R-squared value equals 0 with 24 participants in a multiple regression model with four predictors. Hence, our sample size $(n=35)$ can be deemed appropriate for the present analysis.

\section{Procedure}

Baseline sRPM During the first month of the study, students were provided the SRPM in a group setting, in a small quiet room in their school. All students completed the SRPM without any overt verbalizations recorded (Fox \& Charness, 2010). Next, students were given a random identification number and a password with which to $\log$ in to their individual accounts on the RaiseYourIQ ${ }^{\mathrm{TM}}$ website. Students were taught how to turn on the computer, navigate to the appropriate website, and log on. They were also taught the meanings of English phrases 'same as', 'opposite of', 'more than', and 'less than', which are not easily translated into Bengali (Naskar \& Bandyopadhyay, 2006, p. 90). It took approximately $40 \mathrm{~min}$ to teach each child the meaning of the relational cues. ${ }^{3}$ Once students had completed English instruction training, they underwent the RAI for a

\footnotetext{
${ }^{3}$ Children were pre-trained to derive relations of sameness, opposition and comparison using real objects (two pencils, sheet of paper, two pens, eraser) in the Bengali language. These pre-training trials would involve presenting two objects (e.g., pencil-1, paper) and a cue (the words "OPPOSITE OF" on a sheet of paper) in left to right object>cue>object sequences (e.g., pencil-1 $>$ SAME AS $>$ pen-2, pen- $2>$ OPPOSITE OF $>$ paper). This would be followed by a test sequence (e.g., pencil-1 $>$ OPPOSITE OF>paper) and the question as to whether the test sequence was correct. If Ss could successfully derive relations five SAME/OPPOSITE and five MORE/LESS relations, pre-training was terminated.
} 
baseline assessment of their relating skills. Following completion of the RAI, participants moved on to SMART training.

\section{Relational Ability Training}

Training with the SMART program took place in the school computer laboratory. Training took place with groups of children numbering between 4 and 7 per session. Each participant progressed through the training stages at his/her own pace. Students were partitioned into groups of 2-3 with each student receiving $30 \mathrm{~min}$ of training twice per week for 12 weeks, yielding 24 sessions per student. As noted earlier, the regular attendance and motivation of students could not be ensured given the surrounding political circumstances (Ruud, 2015). Nevertheless, the training sessions were made available to students and they engaged and progressed with the training as much as was possible given their circumstances, with no child skipping more than four sessions (out of 24). All students would therefore undergo a baseline sRPM, followed by SMART training for 3 months, followed by a re-administration of the sRPM 3 months after, regardless of how many stages they were able to complete. In other words, the current study permitted the stages completed to vary based on individual differences while keeping training time constant (also see Hayes \& Stewart, 2016).

Post-sRPM Six months after the baseline sRPM and 3 months after SMART training, the children underwent the sRPM a second time. The second SRPM ranged between 30 and 40 min per participant to complete, taking slightly less time than the baseline assessment. Although it was initially planned that the children would complete the RAI a second time also, the school had lost its computer laboratory at this stage and it was not possible to re-administer the task. Consequently, the only measure that allowed for a pre-post contrast were the Raven's Matrices.

\section{Results}

Individual scores for age, baseline RAI, baseline/pre-sRPM, post-sRPM, pre-post-sRPM differences, last stage completed and change in SRPM performances for individual participants are provided in Tables 1. Participants were ordinally partitioned into four groups based on the number of stages completed for secondary analysis. Specifically, group $1(n=$ 10) consisted of participants who did not progress beyond stage 1; group $2(n=8)$ did not go beyond stages 6 and 7; group $3(n=11)$ showed proficiency with same and opposite relations involving terms no more than one nodal unit apart; finally, group $4(n=6)$ demonstrated partial/complete proficiency with same and opposite relations across terms that were two nodal units apart. These four groups were accordingly
Table 1 Individual performances along the Ravens' matrix (sRPM) and last SMART stage completed

\begin{tabular}{|c|c|c|c|c|c|}
\hline $\mathrm{Age}^{\mathrm{a}}$ & sRPM-pre & sRPM-post & Pre-Post ${ }^{\mathrm{b}}$ & Last stage $^{\mathrm{d}}$ & Group $^{c}$ \\
\hline 10 & 25 & 24 & -1 & 1 & Low \\
\hline 9 & 21 & 23 & 2 & 1 & Low \\
\hline 7 & 31 & 29 & -2 & 1 & Low \\
\hline 13 & 35 & 39 & 4 & 1 & Low \\
\hline 12 & 22 & 24 & 2 & 1 & Low \\
\hline 11 & 25 & 26 & 1 & 1 & Low \\
\hline 11 & 28 & 31 & 3 & 1 & Low \\
\hline 7 & 32 & 32 & 0 & 1 & Low \\
\hline 8 & 20 & 19 & -1 & 1 & Low \\
\hline 12 & 28 & 30 & 2 & 1 & Low \\
\hline 10 & 32 & 32 & 0 & 6 & Med-low \\
\hline 11 & 30 & 34 & 4 & 6 & Med-low \\
\hline 10 & 33 & 32 & -1 & 6 & Med-low \\
\hline 9 & 39 & 39 & 0 & 6 & Med-low \\
\hline 12 & 39 & 39 & 0 & 6 & Med-low \\
\hline 10 & 31 & 31 & 0 & 6 & Med-low \\
\hline 8 & 29 & 28 & -1 & 7 & Med-low \\
\hline 11 & 43 & 47 & 4 & 7 & Med-low \\
\hline 6 & 35 & 36 & 1 & 13 & Med \\
\hline 11 & 34 & 42 & 8 & 14 & Med \\
\hline 7 & 31 & 36 & 5 & 15 & Med \\
\hline 8 & 41 & 45 & 4 & 16 & Med \\
\hline 10 & 28 & 35 & 7 & 17 & Med \\
\hline 9 & 36 & 43 & 7 & 17 & Med \\
\hline 12 & 32 & 35 & 3 & 18 & Med \\
\hline 10 & 34 & 39 & 5 & 20 & Med \\
\hline 8 & 34 & 39 & 5 & 21 & Med \\
\hline 8 & 33 & 37 & 4 & 21 & Med \\
\hline 9 & 27 & 31 & 4 & 22 & Med \\
\hline 10 & 31 & 38 & 7 & 24 & High \\
\hline 13 & 36 & 42 & 6 & 26 & High \\
\hline 8 & 40 & 44 & 4 & 26 & High \\
\hline 11 & 33 & 34 & 1 & 28 & High \\
\hline 9 & 30 & 39 & 9 & 29 & High \\
\hline 10 & 38 & 42 & 4 & 30 & High \\
\hline
\end{tabular}

${ }^{\text {a }}$ Age in months is not available as many of the children were unsure of their birthday and we did not have access to their birth records

${ }^{\mathrm{b}}$ sRPM scores post-SMART training subtracted from sRPM scores acquired previous to SMART training. Negative values indicate that Ss performed poorer on the SRPM after SMART training

${ }^{\mathrm{c}}$ Participants were ordinally ranked based on the stage up to which they could progress

${ }^{\mathrm{d}}$ The last stage a given participant progressed to during SMART

labeled 'low', 'med-low', 'med', and 'high', respectively (again, see Table 1).

Our analyses here consisted of two stages. First, we ran a multiple linear regression to determine whether changes in 
sRPM scores could be predicted by age, baseline RAI, baseline sRPM, and/or the number of SMART stages completed. Second, we conducted an independent samples Kruskal-Wallis $H$ test to determine whether any of the ordinally partitioned groups (see above) varied significantly from one another in relation to the magnitude of pre-post-sRPM differences. As the groups here consisted of unequal sample sizes, they were not included as predictors in the regression.

\section{Multiple Regression Analysis}

An analysis of standard residuals revealed no statistical outliers in the data (Std. residual range: -2.28 to 2.10 ). Multicollinearity was not an issue for age, baseline RAI, baseline sRPM, and last stage completed (tolerances $>.7$ ). The assumption of independent errors was met (Durbin-Watson $=1.66$ ). A histogram of standardized residuals indicated an approximately normal distribution, as did the P-P plot of standardized residuals, which was close to the line. The assumption of non-zero variances was met for age (variance $=3.21$ ), baseline RAI (Var. =23.37), baseline sRPM (Var. =30.05), last stage completed (Var. $=97.73$ ), and change in sRPM (Var. $=8.42$ ). The assumptions for a multiple linear regression were therefore met.

Using the enter method, the four predictor variables explained a significant amount of pre-post sRPM variance, $F$ $(4,30)=7.612, p<.001, R^{2}=.504, R^{2}$ adjusted $=.438, f^{2}=$ 1.02. Computing the degrees of freedom as $\mathrm{N}(35)-\mathrm{K}(4)-1$, analysis of the coefficients revealed that age $(\beta=.230$, $p=.086)$, baseline RAI $(\beta=.219, p=.131)$, and baseline sRPM $(\beta=-.141, p=.364)$ did not significantly predict pre-post-sRPM variance. Only the last stage completed predicted the magnitude of pre-post-sRPM variance, $\beta=.691, t$ $(30)=4.885, p<.001$. Post hoc power analysis with $\alpha=.05$ and $f^{2}=1.02$ demonstrated a high power $(1-\beta)$ of .997 . Keeping $\mathrm{R}$ at chance levels (.5) with $\alpha=.05$ and (1- $\beta$ ) $=.997$, the positive predictive value (PPV; Button et al., 2013) was calculated to be $91 \%$, which is the probability of the regression equation being a true effect.

\section{Correlations Between Pre- and Post-sRPM Outcomes}

A Pearson's product-moment correlation assessed the relationship between SRPM performances prior to, and after, SMART training. Preliminary analyses showed the relationship to be linear with pre-sRPM $(p=.819)$ and post-sRPM ( $p=.661)$ variables normally distributed, as determined by Shapiro-Wilk. A strong positive correlation between preand post-sRPM performances was found, $r(35)=.91$, $p<.001$, with pre-sRPM explaining $82.6 \%$ of the variation in post-sRPM. The high correlation at group level between baseline and post-sRPM performances suggests a test-retest effect, rather than the effects of (often minimal) training.
Running similar correlations across the partitioned groups (i.e., low, med-low, med, high) revealed that pre-sRPM and post-sRPM were significantly $(p<.001)$ correlated across for the low $(r=.94, p<.001)$, med-low $(r=.95, p<.001)$, and medium $(r=.88, p<.001)$ groups. Only the high group was not significantly related with pre-post-sRPM $(r=.73$, $p=.096$ ).

\section{Between-Group Differences in sRPM Performance}

An independent samples Kruskal-Wallis $\mathrm{H}$ test determined whether any of the four groups, partitioned by number of stages completed (Fig. 2), significantly differed from each other in relation to post-sRPM-baseline sRPM differences. Distribution of pre-post SRPM differences was similar for all groups, as assessed by visual inspection of a boxplot. Median difference scores were significantly different between groups, $H(3)=17.93, p<.001$. Bonferroni-corrected pairwise comparisons, using Dunn's (1964) recommendations for adjusting $p$ values, revealed significant differences between low and $\operatorname{high}(p=.037)$, between low and medium $(p=.009)$, between med-low and medium $(p=.010)$, and between med-low and high $(p=.034)$ groups. No significant differences were found between low and med-low, or between med and high groups. Our results indicate that the mean difference scores (between post-sRPM and baseline-sRPM measurements) for those who completed more than 7 training stages $(n=18)$ were significantly greater than those who completed 7 stages or fewer $(n=17)$.

\section{Discussion}

The goal of the present study was to explore whether a program for strengthening mental abilities through relational training, shown to enhance intellectual abilities in populations knowledgeable in English (Cassidy et al., 2011, 2016; Hayes \& Stewart, 2016; Thirus et al., 2016), could be applied to a non-English speaking, socioeconomically disadvantaged cohort with no history of formal education while residing under sub-optimal conditions. Specifically, we asked whether age, baseline relating ability, baseline sRPM performance, and/or the last stage completed could predict changes in SRPM performance. Of the four predictors, only the last SMART stage completed significantly predicted gains in sRPM performance ( $\beta=.691, p<.001)$. Participants were next sectioned into four groups based on the last stage completed, and their pre-post-sRPM gains compared (Table 1). Participants who progressed up to stage 13 and beyond $(n=17)$ produced significantly higher $(p<.001)$ sRPM scores following SMART training than participants who were unable to progress beyond stage 7 . We corroborate the findings reported by Thirus et al. (2016) by showing that SMART performance can predict 
Fig. 2 Differences between preand post-sRPM performances ( $y$ axis) across the four groups ordinally partitioned along last stage completed ( $x$-axis).

Members of the high, medium, and medium-low groups completed between 24 and 30 , 13-22, and 6-7 stages, respectively. Members of the low group did not progress beyond stage 1 (see Table 1). Error bars indicate $1 * \mathrm{SE}$

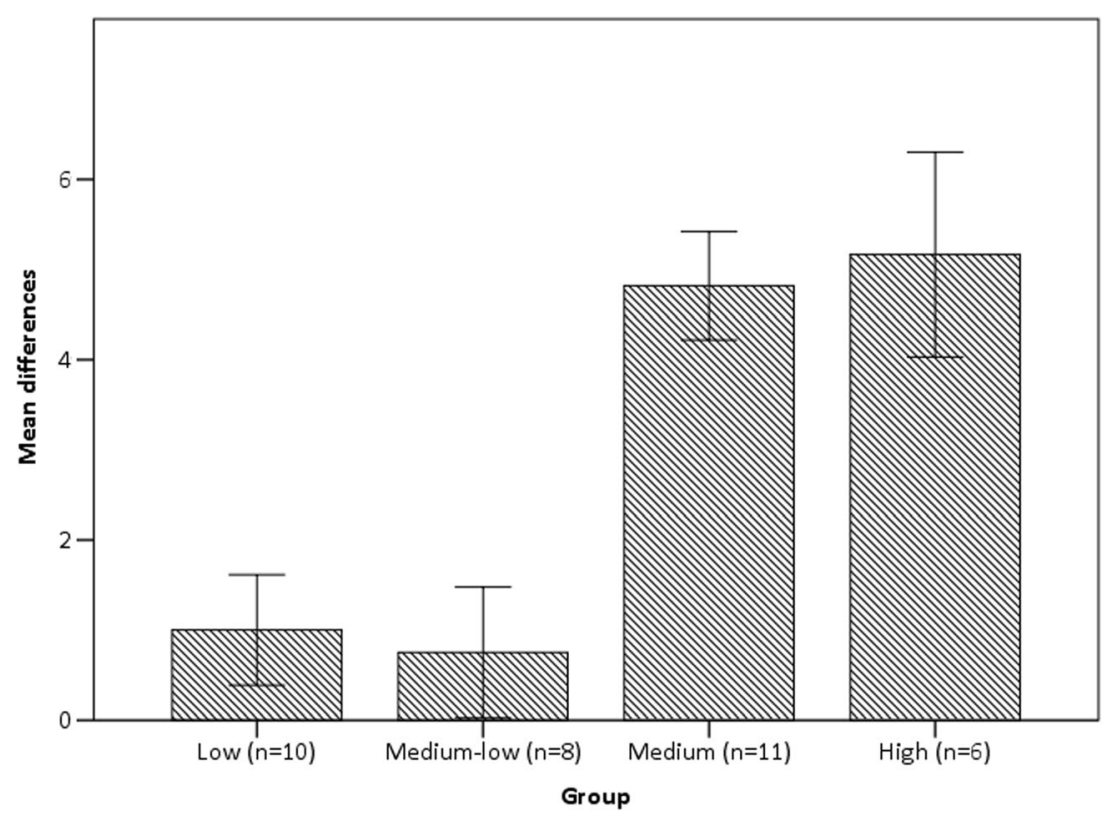

improvements in sRPM performance and, by extension, fluid intelligence. The unique setting of our study further demonstrates that SMART training can be effective for children despite living in poor socio-economic conditions.

Our work adds to the literature on the effects of relational ability training on intelligence (Cassidy et al., 2016; Hayes \& Stewart, 2016; Parra \& Ruiz, 2016; Thirus et al., 2016; Vizcaino-Torres et al., 2015), while also expanding upon previous research in two ways. First, we demonstrate that SMART training can be effective for children who are socioeconomically disadvantaged with little-to-no prior knowledge of English, corroborating the idea that training relational skills per se can enhance performance on other intelligence measures (e.g., Carpenter et al., 1990; Roche et al., 2013; Smoke, 1932). Second, while age, baseline relational skill ability, and baseline fluid intelligence were ruled out as potential predictors of sRPM performance, gender was not. This was because the ratio of females to males in the current sample was disproportionate. Future studies could investigate whether gender may predict sRPM performances (cf., Mackintosh \& Bennett, 2005).

Some limitations of the current study warrant mention. First, when noting the range of inter-individual differences in performances observed here (Table 1), one cannot argue lack of attendance as a significant confound, given that no student skipped more than four sessions out of 24 sessions overall. Rather, some students appeared stuck at a particular stage, which may have reduced motivation in subsequent sessions. Perhaps individual differences in cognitive ability, attention, motivation, and/or other factors may also had influenced the results. At present, it is not possible to address these concerns as the task structure did not permit experimenters to intervene for any of the children in order to keep training conditions consistent. Future work would nevertheless benefit from incorporating personality questionnaires and other self-report instruments to explore the contribution of individual characteristics in SMART performances. Second, given the removal of computer access near the end of the study, it was not possible to administer the RAI a second time to assess for differences in relational responding skills. While one can argue that the last stage completed served as a proxy of relational responding, this cannot compare with the RAI, which is a direct test of the relational skills taught through SMART. Future researchers can include the RAI among other pre-post measures to determine the extent to which the relating skills trained in SMART generalize to other measures of intelligence. Third, given that only one child progressed to more/less training (stage 30 - see Table 1), the results reported here reflect dosage effects of learning increasingly complex same, opposite, and same-opposite combined relations only. A future work could compare the outcomes reported here with that of a matched group who undergoes training along more, less, and more-less combined relations to determine if the present effects are driven by relational training in general (in which case, there should be no difference between the groups), or by the specific type of relations learned (e.g., same/opposite versus more/less). Fourth, when viewing the raw sRPM scores in Table 1, five participants can be observed to have produced a lower sRPM score after SMART training. In response, note that three of those participants did not progress beyond stage 1 , indicating that their dip in sRPM outcome was not likely related to SMART training. The remaining two participants did not advance further than stage 7, which involved deriving whether two terms were the same, or 
opposite, following the presentation of two propositions along opposite relations (Fig. 1). Note that from stage 7, participants were sometimes required to derive across terms one nodal unit apart, which could complicate the derivation required (e.g., opposite + opposite $=$ same, Fig. 1). Stage 7 may reflect those participants' relational 'threshold', that is, the limit of their pre-existing relating repertoire. As stated earlier, subsequent extensions of the current work could examine more closely relationship between the progression individual participants make through SMART and baseline levels of intellectual ability, as assessed through intelligence tests.

Finally, one may ask whether exploring SMART's effectiveness towards the cohort examined here may have confounded with our secondary goal of determining whether level of training provided predicts intellectual performances. While it is true that inter-individual differences and attendance were not controlled for here, the results of Thirus et al. (2016) corroborate with our own, strengthening our conclusion in regard to the effectiveness of SMART. Specifically, note that the top 15 performers in the present study produced greater pre-post-sRPM differences than the treatment group $(n=15)$ in the work reported by Thirius, even though the latter completed all 55 training stages. Specifically, in their study, a gain of 2.1 sRPM points was observed for the treatment group, compared to a gain of 5 points for the top 15 learners in the current study. Perhaps this was due to the lower levels of baseline relational ability of our participants, who likely had more to gain from the earlier stages of training than did the cohort of Thirus et al. (2016). Indeed, the baseline sPRM scores of our top 15 participants was 33.6, which is notably lower than the baseline SRPM of the matched cohort in the Thirus study, which was 51.1. Future research can attempt to investigate the level of relational training at which intellectual gains reach asymptote in order to identify demographics for whom SMART could be most effective.

The present findings further the evidence for the benefits of relational ability training for enhancing intellectual ability in school-aged children, extending the work initiated by Cassidy et al. (2011). While keeping in mind the limitations raised earlier, our results are nevertheless promising given the cohort under investigation and the conditions under which training took place. The child participants in the current study came from impoverished backgrounds with limited exposure to formal curricula. Despite this, many children were still able to achieve a high degree of proficiency in SMART training, which translated to increased fluid intelligence, as implied by pre-post differences in SRPM performances. This suggests that relating skills may consist of the basic behavioral repertoires on which subsequent intellectual abilities develop (Staats, 2012, pp. 178-182). It is hoped that the current findings will inform the development of learning interventions for disadvantaged children, whose access to educational resources are already limited.
Acknowledgements The authors thank Mr. Korvi Rakshand and the JAAGO school staff for their assistance and patience, without whom the current work would not have been possible.

Funding Preparation of this manuscript was funded in part by grant \# 2015/24159-4 from the Sao Paulo Research foundation (FAPESP), as well as from the the Irish Research Council (IRC), to the first author.

\section{Compliance with Ethical Standards}

The current research complied with the ethical and child protection guidelines set forth by the Federal University of Sao Carlos.

Conflict of Interest

In accordance with Springer policy and our ethical obligation as researchers, we declare that the second author is a co-founder, scientific advisor and shareholder of www.RaiseYourIQ.com, which commercially distributes the SMART behavioral intervention examined in the current study. This potential conflict of interest has been disclosed fully to the Editor.

\section{References}

Adey, P., Csapó, B., Demetriou, A., Hautamäki, J., \& Shayer, M. (2007). Can we be intelligent about intelligence? Why education needs the concept of plastic general ability. Educational Research Review, 2(2), 75-97. https://doi.org/10.1016/j.edurev.2007.05.001.

Ainscow, M. (2005). Developing inclusive education systems: What are the levers for change? Journal of Educational Change, 6(2), 109 124. https://doi.org/10.1007/s10833-005-1298-4.

Amd, M., \& Roche, B. (2016). A derived transformation of emotional functions using self-reports, implicit association tests, and frontal alpha asymmetries. Learning \& Behavior, 44(2), 175-190. https:// doi.org/10.3758/s13420-015-0198-6.

Bakhiet, S., Haseeb, B., Seddieg, I., Cheng, H., \& Lynn, R. (2015). Sex differences on raven's standard progressive matrices among 6 to 18 year olds in Sudan. Intelligence, 50, 10-13. https://doi.org/10.1016/ j.intell.2015.01.013.

Blackledge, J. T. (2003). An introduction to relational frame theory: Basics and applications. The Behavior Analyst Today, 3(4), 421433. https://doi.org/10.1037/h0099997.

Burns, G. L., \& Staats, A. W. (1981). Intelligence and child development: What intelligence is and how it is learned and functions. Genetic Psychology Monographs, 104(2), 237-301.

Button, K. S., Ioannidis, J. P. A., Mokrysz, C., Nosek, B. A., Flint, J., Robinson, E. S. J., \& Munafò, M. R. (2013). Power failure: Why small sample size undermines the reliability of neuroscience. Nature Reviews Neuroscience, 14(5), 365-376. https://doi.org/10.1038/ nrn3475.

Carpenter, P. A., Just, M. A., \& Shell, P. (1990). What one intelligence test measures: A theoretical account of the processing in the raven progressive matrices test. Psychological Review, 97(3), 404-431. https://doi.org/10.1037/0033-295X.97.3.404.

Cassidy, S., Roche, B., Colbert, D., Stewart, I., \& Grey, I. M. (2016). A relational frame skills training intervention to increase general intelligence and scholastic aptitude. Learning and Individual Differences, 47, 222-235. https://doi.org/10.1016/j.lindif.2016.03.001.

Cassidy, S., Roche, B., \& Hayes, S. C. (2011). A relational frame training intervention to raise intelligence quotients: A pilot study. The Psychological Record, 61(2), 173-198.

Cassidy, S., Roche, B., \& O'Hora, D. (2010). Relational frame theory and human intelligence. European Journal of Behavior Analysis, 11(1), 37-51. https://doi.org/10.1080/15021149.2010.11434333. 
Cattell, R. B. (1963). Theory of fluid and crystallized intelligence: A critical experiment. Journal of Educational Psychology, 54(1), 122. https://doi.org/10.1037/h0046743.

Cockcroft, K., Alloway, T., Copello, E., \& Milligan, R. (2015). A crosscultural comparison between south african and british students on the wechsler adult intelligence scales third edition (WAIS-III). Frontiers in Psychology, 6, 297. https://doi.org/10.3389/fpsyg. 2015.00297.

Daley, T. C., Whaley, S. E., Sigman, M. D., Espinosa, M. P., \& Neumann, C. (2003). IQ on the rise: The flynn effect in rural kenyan children. Psychological Science, 14(3), 215-219. https://doi.org/10.1111/ 1467-9280.02434.

Di Fabio, A., \& Saklofske, D. (2014). Comparing ability and self-report trait emotional intelligence, fluid intelligence, and personality traits in career decision. Personality and Individual Differences, 64, 174 178. https://doi.org/10.1016/j.paid.2014.02.024.

Dixon, M., Whiting, S., Rowsey, K., \& Belisly, J. (2014). Assessing the relationship between intelligence and the PEAK relational training system. Research in Autism Spectrum Disorders, 8(9), 1208-1213. https://doi.org/10.1016/j.rasd.2014.05.005.

Dunn, O. J. (1964). Multiple comparisons using rank sums. Technometrics, 6(3), 241-252

Faul, F., Erdfelder, E., Lang, A., \& Buchner, A. (2007). GPower 3: A flexible statistical power analysis program for the social, behavioral, and biomedical sciences. Behavior Research Methods, 39(2), 175191. https://doi.org/10.3758/BF03193146.

Fox, M. C., \& Charness, N. (2010). How to gain eleven IQ points in ten minutes: Thinking aloud improves raven's matrices performance in older adults. Aging, Neuropsychology, and Cognition, 17(2), 191204. https://doi.org/10.1080/13825580903042668.

Hayes, S. C., Barnes-Holmes, D., \& Roche, B. (2001). Relational frame theory: A post-skinnerian account of human language and cognition. New York: Kluwer Academic/Plenum Publishers.

Hayes, T., Petrov, A., \& Sederberg, P. (2015). Do we really become smarter when our fluid-intelligence test scores improve? Intelligence, 48, 1-14. https://doi.org/10.1016/j.intell.2014.10.005.

Hayes, J., \& Stewart, I. (2016). Comparing the effects of derived relational training and computer coding on intellectual potential in school-age children. British Journal of Educational Psychology, 86(3), 397-411. https://doi.org/10.1111/bjep.12114.

Jäeggi, S. M., Buschkuehl, M., Jonides, J., \& Shah, P. (2011). Short- and long-term benefits of cognitive training. Proceedings of the National Academy of Science, 108(25), 10081-10086. https://doi.org/10. 1073/pnas.1103228108.

Lovaas, O. I. (1987). Behavioral treatment and normal educational and intellectual functioning in young autistic children. Journal of Consulting and Clinical Psychology, 55(1), 3-9. https://doi.org/10. 1037/0022-006X.55.1.3

Mackintosh, N. J., \& Bennett, E. S. (2005). What do raven's matrices measure? An analysis in terms of sex differences. Intelligence, 33(6), 663-674. https://doi.org/10.1016/j.intell.2005.03.004.

McIlvane, W. J., \& Dube, W. V. (2003). Stimulus control topography coherence theory: Foundations and extensions. The Behavior Analyst, 26(2), 195-213.

Miele, F. (1979). Cultural bias in the WISC. Intelligence, 3(2), 149-163. https://doi.org/10.1016/0160-2896(79)90013-8.

Moran, A. P. (1986). The reliability and validity of raven's standard progressive matrices for irish apprentices. Applied Psychology, 35(4), 533-538. https://doi.org/10.1111/j.1464-0597.1986.tb00955.x.

Moran, L., Stewart, I., McElwee, J., \& Ming, S. (2010). Brief report: The training and assessment of relational precursors and abilities (TARPA): A preliminary analysis. Journal of Autism and Developmental Disorders, 40(9), 1149-1153. https://doi.org/10. 1007/s10803-010-0968-0.

Naskar, S. K., \& Bandyopadhyay, S. (2006). Handling of prepositions in English to Bengali machine translation. In Proceedings of the Third
ACL-SIGSEM Workshop on Prepositions (pp. 89-94). Association for Computational Linguistics, Trento, Italy, April 6. East Stroudsburg, PA.

O'Toole, C., Barnes-Holmes, D., Murphy, C., O'Connor, J., \& BarnesHolmes, Y. (2009). Relational flexibility and human intelligence: Extending the remit of skinner's verbal behavior. International Journal of Psychology and Psychological Therapy, 9(1), 1-17.

Owen, K. (1992). The suitability of raven's standard progressive matrices for various groups in south africa. Personality and Individual Differences, 13(2), 149-159. https://doi.org/10.1016/0191-8869 (92)90037-P

Parra, I., \& Ruiz, F. J. (2016). The effect on intelligence quotient of training fluency in relational frames of coordination. International Journal of Psychology and Psychological Therapy, 16(1), 1-12.

Raven, J. (2000). The raven's progressive matrices: Change and stability over culture and time. Cognitive Psychology, 41(1), 1-48. https:// doi.org/10.1006/cogp.1999.0735.

Raven, J. C., \& Court, J. H. (1993). Manual for raven progressive matrices and vocabulary scales by JC raven, JH court and J. Raven; section 2; Coloured progressive matrices. Oxford: Psychologist Press.

Roche, B., Cassidy, S., \& Stewart, I. (2013). Nurturing genius: Using relational frame theory to address a foundational aim of psychology. In T. Kashdan \& J. Ciarrochi (Eds.), Cultivating well-being: Treatment innovations in positive psychology, acceptance and commitment therapy, and beyond (pp. 267-302). Oakland, CA: New Harbinger.

Rohde, T. E., \& Thompson, L. A. (2007). Predicting academic achievement with cognitive ability. Intelligence, $35(1), 83-92$. https://doi. org/10.1016/j.intell.2006.05.004.

Ruud, A. E. (2015). Five scenarios for Bangladesh. Retrieved from http:// archive.dhakatribune.com/long-form/2015/feb/24/five-scenariosbangladesh.

Sefcek, J. A., Miller, G. F., \& Figueredo, A. J. (2016). Development and validation of an 18-item medium form of the ravens advanced progressive matrices. SAGE Open, 6(2), 215824401665191. https://doi. org/10.1177/2158244016651915.

Smoke, K. L. (1932). An objective study of concept formation. Psychological Monographs, 42(4), i-46. https://doi.org/10.1037/ h0093297.

Staats, A. W. (1971). Child learning, intelligence, and personality: Principles of a behavioral interaction approach. New York, NY: Harper \& Row.

Staats, A. W. (2012). The marvelous learning animal: What makes human behavior unique. Amherst, NY: Prometheus Books.

Sternberg, R. J. (1988). The raising of intelligence: A selected history of attempts to raise retarded intelligence. University of Illinois Press., 101, 142. https://doi.org/10.2307/1422800.

Sternberg, R. J. (2008). Increasing fluid intelligence is possible after all. Proceedings of the National Academy of Science, 105, 6791-6792. https://doi.org/10.1073/pnas.0803396105.

te Nijenhuis, J., Bakhiet, S. F., van den Hoek, M., Repko, J., Allik, J. Žebec, M. S., Sukhanovskiy, V., \& Abduljabbar, A. S. (2016). Spearman's hypothesis tested comparing sudanese children and adolescents with various other groups of children and adolescents on the items of the standard progressive matrices. Intelligence, 56, 4657. https://doi.org/10.1016/j.intell.2016.02.010.

Thirus, J., Starbrink, M., \& Jansson, B. (2016). Relational frame theory, mathematical and logical skills: A multiple exemplar training intervention to enhance intellectual performance. International Journal of Psychology and Psychological Therapy, 16(2), 141-155.

Valencia, R. R. (1984). Reliability of the raven coloured progressive matrices for anglo and for Mexican-American children. Psychology in the Schools, 21(1), 49-52. https://doi.org/10.1002/ 1520-6807(198401)21:1<49::AID-PITS2310210109>3.0.CO;2-H.

Vizcaino-Torres, R., Ruiz, F., Luciano, C., Lopez-Lopez, J., BarberoRubio, A., \& Gil, E. (2015). The effect of relational training on intelligence quotient: A case study. Psicothema, 27(2), 120-127. https://doi.org/10.7334/psicothema2014.149. 